\title{
Cryptococcal meningitis complicating remote skull fracture
}

\author{
FR A N CES T. LESTER \\ From the Department of Medicine, Yekatit 12 Hospital, Addis Ababa, Ethiopia
}

SUMMARY A patient in whom cryptococcal meningitis complicated a nine year old depressed frontal skull fracture, an association which has not been reported previously, is recorded. This is also the first case of cryptococcal meningitis recognised in Ethiopia.

Cryptococcal meningitis has been recognised infrequently in African countries, but in recent years 24 cases have been reported in black South Africans (Pillay and Simjee, 1976), and at least five patients have been treated in Nairobi, Kenya (Stewart, 1977). The first case of cryptococcal meningitis recognised in Ethiopia, which occurred in a patient with an old depressed skull fracture, an association not previously recorded, is presented here.

\section{Case report}

In 1968, a 31 year old carpenter (KS) sustained a depressed right frontal skull fracture while separating two combatants. In 1971, he was admitted to hospital with fever and stiff neck, no cause for which was identified. In 1970, 1972, and 1974 he had three documented episodes of acute bacterial meningitis, the last known to be caused by Diplococcus pneumoniae, each of which responded quickly to high doses of penicillin. Between hospitalisations he was mentally normal, had no seizures, and worked regularly, but there was deafness after the 1974 episode. He was not diabetic, and had had no other illnesses. Surgeons were consulted in 1974 because a dural tear was suspected, but they were unwilling to interfere operatively.

In February 1977, at age 40 years, he was again admitted to hospital with seven days of fever and confusion. He was stuporous with fixed, dilated right pupil, normal optic fundi, and a stiff neck. The first spinal fluid examination showed 275 white blood cells $/ \mathrm{mm}^{3}, 56 \%$ of them lymphocytes, and $1.1 \mathrm{mmol} / 1$ of sugar. Culture was not poss-

\footnotetext{
Address for reprint requests: Dr Frances T. Lester, Box 30432, Addis Ababa, Ethiopia.

Accepted 8 February 1978
}

ible. Radiography of the skull showed the old depressed skull fracture, and he was treated, on a presumptive diagnosis of recurrent acute bacterial meningitis, with intravenous penicillin and chloramphenicol in high doses.

He failed to improve, remaining febrile and stuporous. The right pupil remained dilated, and during the second week he was thought, on clinical grounds, to have a cerebral abscess.

The cerebrospinal fluid on the thirteenth day was cloudy, under pressure, with white blood cell count of $1375 / \mathrm{mm}^{3}$ ( $81 \%$ lymphocytes), protein of $0.85 \mathrm{~g} / \mathrm{l}$, low glucose, negative Gram stain, and no growth on culture.

On account of the negative culture, an Indian ink preparation was examined. It showed typical Cryptococcus neoformans organisms with thick capsules. Attempts to culture the fungus were unsuccessful.

The patient was still critically ill, stuporous, febrile, and tachypnoeic, with stiff neck, normal fundi, dilated right pupil, upgoing left plantar response, and bedsores.

Intravenous therapy with amphotericin B was begun immediately after the Indian ink stain was found positive: $195 \mathrm{mg}$ was given over the next 11 days in slow intravenous drips: (5-fluorocytosine is not available in Ethiopia). He began to improve, the neck stiffness disappeared, and on the sixth day of treatment he was able to take fluids and to recognise his family. On the twentysecond hospital day his cerebrospinal fluid was clear with only seven white blood cells $/ \mathrm{mm}^{3}$ and a protein level of $0.28 \mathrm{~g} / 1$. The Indian ink preparation was still positive for Cryptococcus neoformans.

However, his blood urea, which had been normal $(9.9 \mathrm{mmol} / \mathrm{l})$ initially, began to rise, the dose of amphotericin B had to be decreased, and on 672 
the twenty-fifth day he began to deteriorate again. There were no clinical signs of meningitis at this time, but he died on the twenty-sixth day with high fever $\left(40.5^{\circ} \mathrm{C}\right)$, hypotension, infected bedsores, and blood urea level of $34.2 \mathrm{mmol} / 1$. Necropsy was not performed.

\section{Discussion}

Post-traumatic meningitis is a well-recognised syndrome (Hand and Sanford, 1970), and patients may have as many as five recurrences. The prognosis is usually relatively good, the most common infecting organism is $D$. pneumoniae, as in this patient's 1974 episode, and no references to fungi as the infecting agent were found.

Cryptococcus neoformans has a "special predilection for the immunodeficient host," and about half the patients have serious underlying disease, especially lymphomas and long-term steroid therapy (Case Records, 1976). However, this infection appears not to have been reported in association with the recurrent meningitis sometimes seen after skull fractures, which were not mentioned as a predisposing cause for cryptococcal meningitis in reports covering 170 patients (Diamond and Bennett, 1974; Grover et al., 1975; Berger and Paz, 1976; Case records, 1976; Pillay and Simjee, 1976).

Cryptococcus neoformans has been isolated from soil throughout the world especially from the excreta of pigeons and birds (Case records, 1976). However, almost no cases of cryptococcal meningitis had been reported from the African continent until Pillay and Simjee (1976) reported 24 cases in Black South Africans. The apparent rarity in African countries is probably because physicians in understaffed hospitals with poor facilities do not look for the disease: our patient went undiagnosed for two weeks in hospital because the possibility of a fungal meningitis was not considered. The Indian ink preparation can be done easily with a microscope and simple materials: it was positive in 19 of 27 confirmed cases, in 17 of the 19 on the first attempt (Sarosi et al., 1969), and may be positive even if culture is unsuccessful (Berger and Paz, 1976) as in this patient. The demonstration of $C$. neoformans by Indian ink stain, even if culture is negative or impossible, is sufficient justification for therapy (Pillay and Simjee, 1976). This patient is the first case of cryptococcal meningitis to have been recognised in Ethiopia.

The assistance of Dr Messele Gedebou and Ato Tesfaye Akalue of the Bacteriology Department of Addis Ababa University is gratefully acknowledged.

\section{References}

Berger, M. P., and Paz, J. (1976). Diagnosis of cryptococcal meningitis. Journal of the American Medical A ssociation, 236, 2517-2518.

Case Records of the Massachusetts General Hospital, 27-1976. (1976). New England Journal of Medicine, 295, 34-42.

Diamond, R. D., and Bennett, J. E. (1974). Prognostic factors in cryptococcal meningitis. Annals of Internal Medicine, 80, 176-180.

Grover, S., Sharma, K. D., and Jagtap, P. (1975). Cryptococcal meningitis. Journal of Postgraduate Medicine, 21, 151-156.

Hand, W. L., and Sanford, J. P. (1970). Post traumatic bacterial meningitis. Annals of Internal Medicine, 72, 869-874.

Pillay, N., and Simjee, A. E. (1976). Cryptococcal meningitis: our experience in 24 black patients. South African Medical Journal, 50, 1604-1606.

Sarosi, G. A., Parker, J. D., Doto, I. L., and Tosh, F. E. (1969). Amphotericin B in cryptococcal meningitis. Annals of Internal Medicine, 71, 10791087.

Stewart, J. D. (1977). Cryptococcal meningitis treated with 5-fluorocytosine and amphotericin-B. East African Medical Journal. In press. 